\title{
SOME COMPLETELY MONOTONIC FUNCTIONS OF POSITIVE ORDER
}

\author{
STAMATIS KOUMANDOS AND MARTIN LAMPRECHT
}

\begin{abstract}
We completely determine the set of $(\alpha, \beta) \in \mathbb{R}^{2}$ for which the function $\frac{e^{\alpha x}-e^{\beta x}}{e^{x}-1}$ is convex on $(0, \infty)$ and use this result to give some special classes of completely monotonic functions of positive order related to gamma and psi functions.
\end{abstract}

\section{INTRODUCTION}

Euler's gamma function $\Gamma(x)$ is defined by $\Gamma(x)=\int_{0}^{\infty} t^{x-1} e^{-t} d t$ for $x>0$. Its logarithmic derivative $\psi(x)=\Gamma^{\prime}(x) / \Gamma(x)$ is called the psi or digamma function, and the derivatives $\psi^{(n)}(x)$ are called polygamma functions. In this paper we shall extend and strengthen some of the results obtained in 4 regarding inequalities for ratios of gamma functions and differences of digamma and polygamma functions. For background information and an extensive bibliography concerning such inequalities, we refer to [4].

Many of the inequalities presented in [4] are obtained by verifying the complete monotonicity of certain functions. A function $f:(0, \infty) \rightarrow \mathbb{R}$ is said to be completely monotonic if it has derivatives of all orders and satisfies

$$
(-1)^{n} f^{(n)}(x) \geq 0 \text {, for all } x>0 \text { and } n \geq 0 .
$$

J. Dubourdieu [1] proved that if a nonconstant function $f$ is completely monotonic, then strict inequality holds in (1.1). See also 2 for a simpler proof of this result. A necessary and sufficient condition for complete monotonicity is given by Bernstein's theorem (see [11, p. 161]), which states that $f$ is completely monotonic on $(0, \infty)$ if and only if

$$
f(x)=\int_{0}^{\infty} e^{-x t} d \mu(t)
$$

where $\mu$ is a nonnegative measure on $[0, \infty)$ such that the integral converges for all $x>0$.

In [6], Koumandos and Pedersen called a function $f$ completely monotonic of order $n=0,1,2, \ldots$ if $x^{n} f(x)$ is completely monotonic on $(0, \infty)$. Thus, completely monotonic functions of order 0 are the classical completely monotonic functions,

Received by the editor May 7, 2009 and, in revised form, June 16, 2009.

2010 Mathematics Subject Classification. Primary 33B15; Secondary 26D20, 26 D15.

Key words and phrases. Gamma function, psi function, completely monotonic functions.

The research for this paper has been supported by the Leventis Foundation (Grant no. 341121041).

The authors would like to thank Setsuo Takato for his help with KETpic.

(C)2009 American Mathematical Society Reverts to public domain 28 years from publication 
while completely monotonic functions of order 1 are the strongly completely monotonic functions that have been introduced in [10]. It is easy to see that a function $f$ is completely monotonic if $x f(x)$ is completely monotonic, and therefore a function that is completely monotonic of order $n$ is completely monotonic of order $m=0,1, \ldots, n-1$.

In [6, Thm. 1.3], it is furthermore shown that a function $f$ is completely monotonic of order $n \geq 1$ on $(0, \infty)$ if, and only if,

$$
f(x)=\int_{0}^{\infty} e^{-x t} p(t) d t
$$

where the integral converges for all $x>0$ and where $p$ is $n-1$ times differentiable on $[0, \infty)$ with $p^{(n-1)}(t)=\mu([0, t])$ for some Radon measure $\mu$ and $p^{(k)}(0)=0$ for $0 \leq k \leq n-2$. This has already been proven for the case $n=1$ in [10, Thm. 1] and for the case $n=2$ in [4, Lem. 2] and will be used here in the case $n=3$ in order to strengthen some of the results obtained in [4].

The applications of [4, Lem. 2] that are presented in [4] lead to the question for which $(\alpha, \beta) \in \mathbb{R}^{2}$ the function $f_{\alpha, \beta}(x)$, defined by

$$
f_{\alpha, \beta}(x):=\frac{e^{\alpha x}-e^{\beta x}}{e^{x}-1} \quad \text { for } \quad x \in \mathbb{R} \backslash\{0\} \quad \text { and } \quad f_{\alpha, \beta}(0)=\alpha-\beta,
$$

is convex in $(0, \infty)$. We will give a complete solution to this question and thus extend some further results from [4].

In order to state our results, for $\alpha, \beta \in \mathbb{R}$ set

$$
\begin{aligned}
& \varepsilon_{1}(\alpha, \beta):=2 \alpha \beta+2 \alpha^{2}-3 \alpha+2 \beta^{2}-3 \beta+1, \\
& \varepsilon_{2}(\alpha, \beta):=4 \alpha^{2} \beta^{2}-4 \alpha^{2} \beta-4 \alpha \beta^{2}+4 \alpha \beta-\alpha^{2}+\alpha-\beta^{2}+\beta, \\
& \varepsilon_{3}(\alpha, \beta):=\left(\alpha-\frac{1}{2}\right)^{2}+\left(\beta-\frac{1}{2}\right)^{2}-\frac{1}{2},
\end{aligned}
$$

and

$$
\begin{aligned}
& \Gamma_{1}:=\{(\alpha, \beta): 0 \leq \beta \leq 1<\alpha\} \cap\left\{(\alpha, \beta): \varepsilon_{1}(\alpha, \beta)=0\right\}, \\
& \Gamma_{2}:=\{(\alpha, \beta): 0 \leq \beta \leq 1 \leq \alpha\} \cap\left\{(\alpha, \beta): \varepsilon_{2}(\alpha, \beta)=0\right\}, \\
& \Gamma_{3}:=\left\{(\alpha, \beta): \beta \leq \frac{1}{2}-\left|\alpha-\frac{1}{2}\right|\right\} \cap\left\{(\alpha, \beta): \varepsilon_{1}(\alpha, \beta)=0\right\},
\end{aligned}
$$

and let $C$ and $D$ be the open bounded sets whose boundaries are given by the Jordan curves $\Gamma_{1} \cup \Gamma_{2} \cup\left\{(1, \beta): \frac{1}{2} \leq \beta \leq 1\right\}$ and $\Gamma_{2} \cup \Gamma_{3} \cup\left\{(\alpha, \alpha): \frac{1}{6}(3-\sqrt{3}) \leq \alpha \leq 1\right\}$, respectively. Let $H$ denote the half-plane $\{(\alpha, \beta): \beta \leq \alpha\}$ and set

$$
A:=\left(H \cup\left\{(\alpha, 1): 0 \leq \alpha \leq \frac{1}{2}\right\}\right) \backslash D
$$

and $B:=D \backslash\left(C \cup\left\{(1, \beta): 0 \leq \beta \leq \frac{1}{2}\right\}\right)$. (Cf. Figure 1 The graphs in this paper have been created by using the KETpic package for Maple [3.)

It is perhaps interesting to note that the set $\left\{(\alpha, \beta): \varepsilon_{1}(\alpha, \beta)=0\right\}$ describes an ellipse with center $\left(\frac{1}{2}, \frac{1}{2}\right)$ and semi-axes $\frac{1}{\sqrt{2}}$ and $\frac{1}{\sqrt{6}}$ with the major axis forming an angle of $-\frac{\pi}{4}$ with the $\alpha$-axis. $\Gamma_{1}$ and $\Gamma_{3}$ are therefore elliptical arcs $\left(\Gamma_{3}\right.$ is even a quarter-ellipse).

Continuing with necessary definitions, for $\alpha, \beta \in \mathbb{R}$ and $t>0$, define $g_{\alpha, \beta}(t):=$ $g_{\alpha}(t)-g_{\beta}(t)$, where

$$
g_{\alpha}(t):=t^{\alpha-1}\left[(1-\alpha)^{2} t^{2}+\left(1+2 \alpha-2 \alpha^{2}\right) t+\alpha^{2}\right]-t-1,
$$




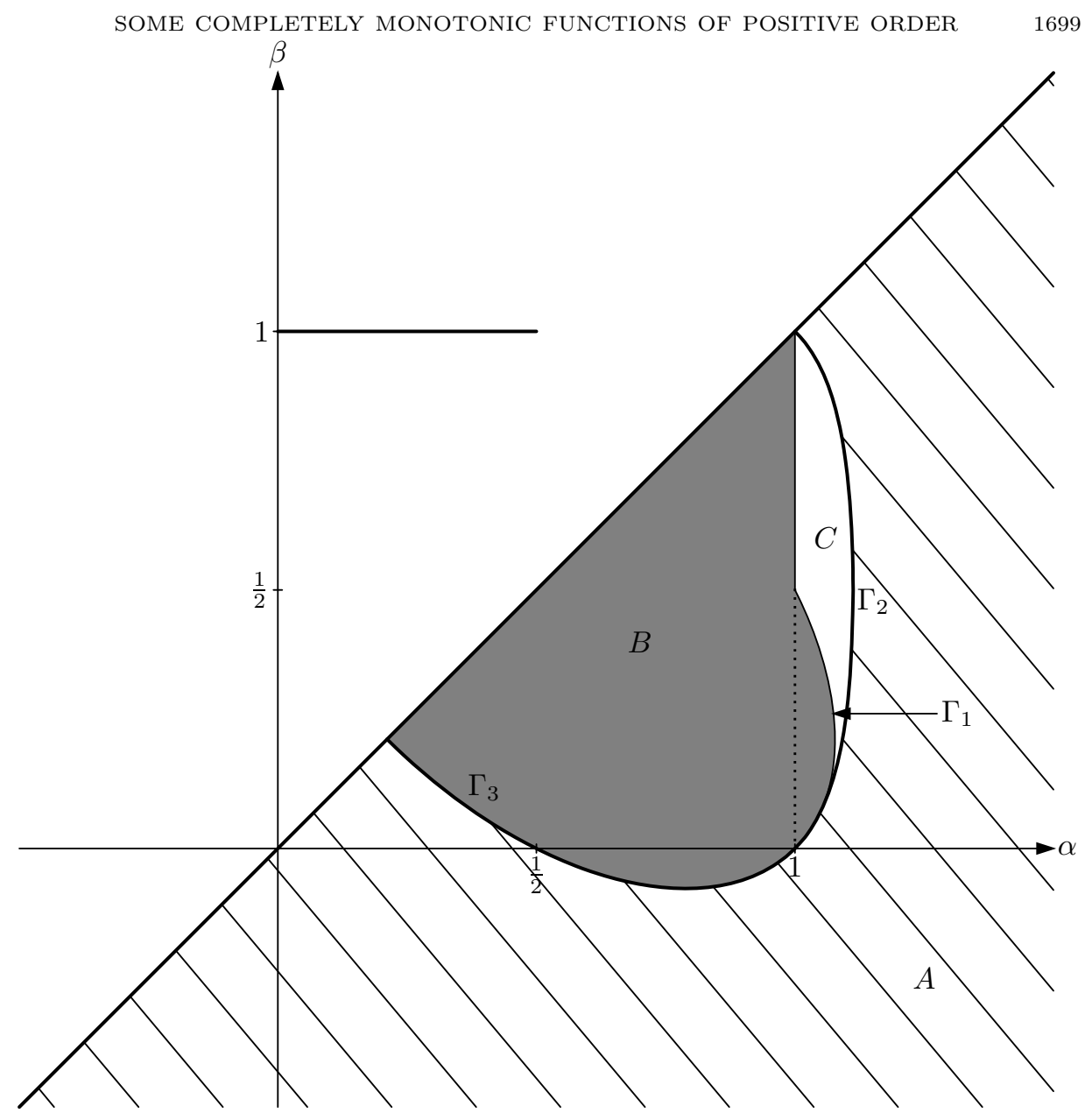

Figure 1 . The sets $A, B$ and $C$. The bold curves are $\partial A$. Note that $\Gamma_{1} \cap \Gamma_{2} \cap \Gamma_{3}=(1,0)$ and that the dotted line $\{(1, \beta): 0<$ $\beta \leq \frac{1}{2}$ \} belongs to neither $A, B$ or $C$, but to $A^{*}$.

and, for $\alpha, \beta \in \mathbb{R} \backslash\{1\}$ with $\varepsilon_{2}(\alpha, \beta) \varepsilon_{3}(\alpha, \beta) \leq 0$,

$$
t^{*}(\alpha, \beta)=\frac{\varepsilon_{2}(\alpha, \beta)-2 \alpha \beta(1-\alpha)(1-\beta)+\sqrt{-\varepsilon_{2}(\alpha, \beta) \varepsilon_{3}(\alpha, \beta)}}{2(1-\alpha)^{2}(1-\beta)^{2}} .
$$

Finally, for any set $M \subset \mathbb{R}^{2}$, let $M^{*}$ denote its reflection with respect to the straight line $\{(\alpha, \alpha): \alpha \in \mathbb{R}\}$.

Theorem 1.1. (1) For $(\alpha, \beta) \in A$ the function $f_{\alpha, \beta}(x)$ is convex in $(0, \infty)$ and for $(\alpha, \beta) \in A^{*}$ it is concave there.

(2) For $(\alpha, \beta) \in B \cup B^{*}$ the function $f_{\alpha, \beta}^{\prime \prime}(x)$ changes sign in $(0, \infty)$.

(3) In $C \cup C^{*}$ the sign of $f_{\alpha, \beta}^{\prime \prime}(x)$ is constant in $(0, \infty)$ if, and only if, $(\alpha, \beta) \in$ $C_{\text {conv }} \cup C_{\text {conv }}^{*}$, where

$$
C_{\text {conv }}:=\left\{(\alpha, \beta) \in C: g_{\alpha, \beta}\left(t^{*}(\alpha, \beta)\right) \geq 0\right\} .
$$

$f_{\alpha, \beta}(x)$ is convex in $(0, \infty)$ if $(\alpha, \beta) \in C_{\text {conv }}$ and concave if $(\alpha, \beta) \in C_{\text {conv }}^{*}$ (cf. Figure 2). 


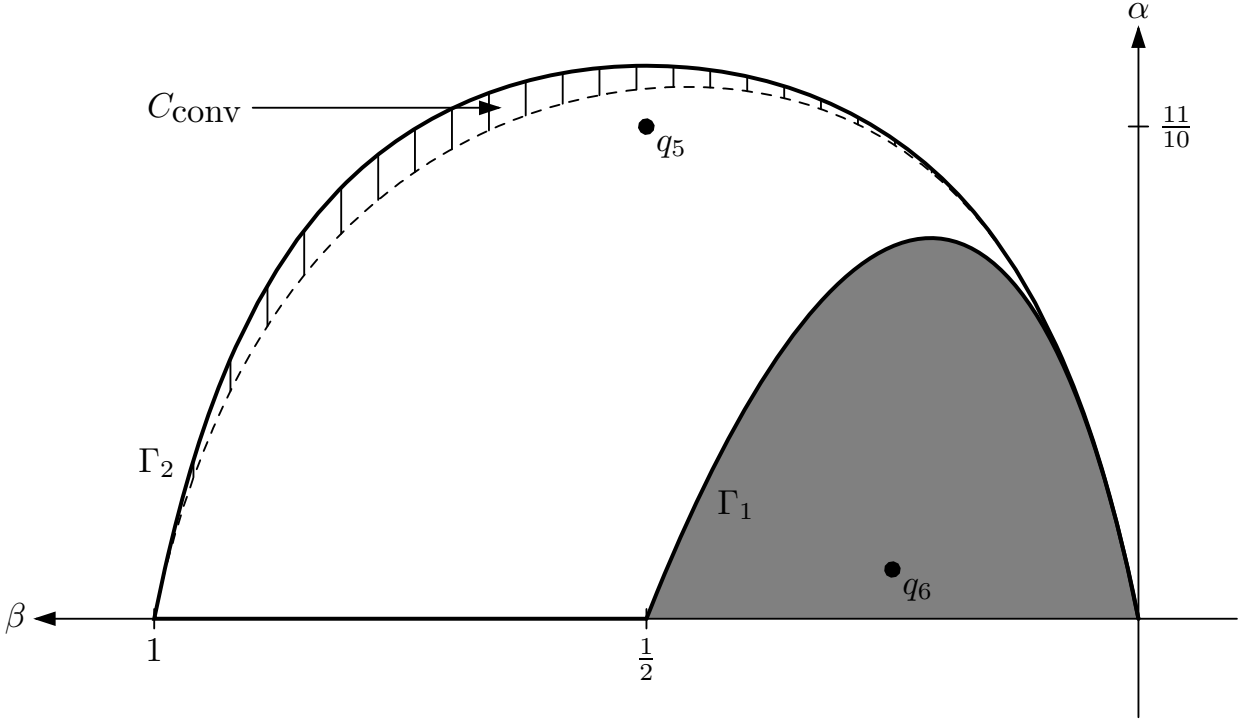

Figure 2. The set $C$. The bold curves are $\partial C$. The dashed curve describes the set of points $(\alpha, \beta) \in C$ for which $g_{\alpha, \beta}\left(t^{*}(\alpha, \beta)\right)=0$.

Let $S_{1}$ denote the set of $(s, t) \in \mathbb{R}^{2}$ such that $(1-t, 1-s) \in A \cup C_{\text {conv }}$ and set

$$
T:=(\{(s, t): 0 \leq s, t\} \cup\{(s, t): t \leq 1-s\}) \backslash\{(s, t): 0<t<1-s<1\}
$$

and $S_{2}:=S_{1} \cap T$.

Theorem 1.1 will allow us to prove the following extensions of results from [4].

Theorem 1.2. (1) Let

$$
L(x):=x-\frac{\Gamma(x+t)}{\Gamma(x+s)} x^{s-t+1} .
$$

Then

$$
\Phi(x):=-\frac{\Gamma(x+s)}{\Gamma(x+t)} x^{t-s-1} L^{\prime \prime}(x)
$$

is completely monotonic of order 2 on $(0, \infty)$ for all $(s, t) \in S_{1}$, and for $(s, t) \in S_{2}$ the function $L^{\prime}(x)$ is completely monotonic on $(0, \infty)$. In particular, for $(s, t) \in S_{2}$, the function $L(x)$ is strictly increasing and concave on $(0, \infty)$ and the inequality

$$
0<x-\frac{\Gamma(x+t)}{\Gamma(x+s)} x^{s-t+1}<\frac{1}{2}(s-t)(s+t-1)
$$

holds for all $x>0$ (cf. [4, Thm. 1]).

(2) For $(s, t) \in S_{2}$ the inequality

$$
\psi(x+t)-\psi(x+s)+\frac{s-t+1}{x}<\frac{\Gamma(x+s)}{\Gamma(x+t)} x^{t-s-1}
$$

holds for all $x>0$ and the function

$$
\psi(x+s)-\psi(x+t)-\frac{s-t}{x}+\frac{(s-t)(s+t-1)}{2 x^{2}}
$$

is completely monotonic in $(0, \infty)$ for all $(s, t) \in S_{1}$ (cf. [4, Cor. 1]). 
(3) For $m, n \in \mathbb{N}$ with $m>n$, let

$$
U_{n, m}(x):=\sum_{k=n}^{m} \frac{(t)_{k}}{(s)_{k}} e^{i k x}, \quad V_{n, m}(x):=\frac{\Gamma(s)}{\Gamma(t)} \sum_{k=n}^{m} \frac{1}{k^{s-t}} e^{i k x},
$$

where $(a)_{k}=a(a+1) \cdots(a+k-1)$. If $(s, t) \in S_{2} \cap\{(s, t): s \geq 1\}$, then for $\frac{\pi}{n} \leq x<\pi, n>1$, the estimate

$$
\left|U_{n, m}(x)-V_{n, m}(x)\right|<\frac{1}{n^{s-t}} \frac{\Gamma(s)}{\Gamma(t)} \frac{(s-t)(s+t-1)}{2}
$$

holds (cf. 4, Prop. 1]).

(4) Let

and

$$
\Lambda(x):=x \log \left(\frac{\Gamma(x+t)}{\Gamma(x+s)} x^{s-t}\right)
$$

$$
K(x):=\psi^{\prime}(x+t)-\psi^{\prime}(x+s)+\frac{2}{x}[\psi(x+t)-\psi(x+s)]+\frac{s-t}{x^{2}} .
$$

If $(s, t) \in S_{1}$, then the function $K(x)=\frac{1}{x} \Lambda^{\prime \prime}(x)$ is completely monotonic of order 2 on $(0, \infty)$ and the function $-\Lambda^{\prime}(x)$ is completely monotonic on $(0, \infty)$. In particular, the function $\Lambda(x)$ is strictly decreasing and convex on $(0, \infty)$, so that

$$
-\frac{(s-t)(s+t-1)}{2}<x \log \left(\frac{\Gamma(x+t)}{\Gamma(x+s)} x^{s-t}\right)<0, \text { for all } x>0 .
$$

In particular, when $s=0$, then the above results hold for $t \leq 0$ and $\frac{1}{2} \leq$ $t \leq 1$, but not for any other $t \in \mathbb{R}$ (cf. [4, Prop. 2]).

Special cases of Theorem 1.2 (3) were the main tools in [7] and [8] for the estimation of certain trigonometric sums arising in the context of starlike functions. Moreover, it is perhaps interesting to note that in 5 the exact range of $t$ for which inequality (1.2) holds when $s=1$ has been determined to be $\left[\frac{1}{3}, 1\right)$.

An application of [6. Thm. 1.3] leads to the following.

Theorem 1.3. (1) There is an analytically defined $a^{*}<0$ with numerical value $a^{*}=-0.0741 \ldots$, such that the function

$\xi(x)=a^{2}\left[\psi^{\prime}(x)\right]^{2}+a \psi^{\prime \prime}(x)+\frac{2 a(1-a)}{x} \psi^{\prime}(x)-\frac{a(1-a)}{x^{2}}, \quad a \neq 0$,

is completely monotonic of order 3 in $(0, \infty)$ if, and only if, $a \in\left(-\infty, a^{*}\right] \cup$ $\left[\frac{2}{3}, \infty\right)(c f$. [4, Thm. $\left.2(1)]\right)$.

(2) The functions

$$
\begin{aligned}
f_{1}(x) & :=\left[\psi^{\prime}(x)\right]^{2}+\psi^{\prime \prime}(x) \\
f_{2}(x) & :=\frac{2}{3}\left(\psi^{\prime}(x)+\frac{1}{2 x}\right)^{2}+\psi^{\prime \prime}(x)-\frac{1}{2 x^{2}}
\end{aligned}
$$

are completely monotonic of order 3 on $(0, \infty)$. On the other hand, while being completely monotonic of order 2 , the function

$$
f_{3}(x)=-\psi^{\prime \prime}(x)-\frac{2}{x} \psi^{\prime}(x)+\frac{1}{x^{2}}
$$

is not completely monotonic of order 3 on $(0, \infty)$ (cf. [4, Cor. 3]). 


\section{Proofs of Theorems 1.2 and 1.3}

We shall first show that in the case $s=0$ the function $K$ defined in Theorem 1.2 (4) is not completely monotonic for $t \in\left(0, \frac{1}{2}\right) \cup(1, \infty)$. To that end observe that by the asymptotic formula

$$
\psi(x+t)-\psi(x)=\frac{t}{x}-\frac{t(t-1)}{2 x^{2}}+\frac{t\left(1-3 t+2 t^{2}\right)}{6 x^{3}}+O\left(x^{-4}\right), \quad x \rightarrow \infty,
$$

we have

$$
6 x^{4} K(x) \rightarrow-2 t^{3}+3 t^{2}-t, \quad x \rightarrow \infty .
$$

Since $-2 t^{3}+3 t^{2}-t$ takes negative values for $t \in\left(0, \frac{1}{2}\right) \cup(1, \infty), K$ cannot be completely monotonic on $(0, \infty)$ for those $t$.

The remaining statements of Theorem 1.2 follow from the proofs of Thm. 1, Cor. 1, Prop. 1 and Prop. 2 in [4, since $f_{1-t, 1-s}(x)$ is convex on $(0, \infty)$ for all $(s, t) \in S_{1}$ by Theorem 1.1 and convex and monotonic on $(0, \infty)$ for all $(s, t) \in S_{2}$ by Theorem 1.1 and 9 , Thm. $1.1(3),(4)]$.

In order to prove Theorem 1.3 (1) note that in the proof of [4, Thm. 2] it is shown that $\xi$ can be written as the Laplace transform of the function

$$
G_{a}(u):=a^{2} \int_{0}^{u} \delta(u-v) \delta(v) d v+2 a(1-a) \int_{0}^{u} \delta(v) d v-a u \delta(u)-a(1-a) u,
$$

where

$$
\delta(u):=\frac{u e^{u}}{e^{u}-1}, \quad \delta(0):=1 .
$$

In the proof of [4, Thm. 2] it is also shown that $G_{a}^{(n)}(u) \geq 0$ for $n=0,1,2, u>0$ and $a \in(-\infty, 0] \cup\left[\frac{2}{3}, \infty\right)$ and that

$$
G_{a}^{\prime \prime}(u)=a^{2} \int_{0}^{u} \delta^{\prime}(u-v) \delta^{\prime}(v) d v-a u \delta^{\prime \prime}(u) .
$$

Hence, it follows from [6, Thm. 1.3] that $\xi(x)$ will be completely monotonic of order 3 on $(0, \infty)$ if, and only if, $G_{a}^{\prime \prime \prime}(u) \geq 0$ for $u \geq 0$. From the above formula for $G_{a}^{\prime \prime}(u)$ we calculate

$$
G_{a}^{\prime \prime \prime}(u)=a^{2}\left(\int_{0}^{u} \delta^{\prime \prime}(u-v) \delta^{\prime}(v) d v+\frac{\delta^{\prime}(u)}{2}\right)-a\left(u \delta^{\prime \prime \prime}(u)+\delta^{\prime \prime}(u)\right) .
$$

From the proof of [4, Thm. 2] we obtain

$$
\delta^{\prime}(u)>0, \quad \delta^{\prime \prime}(u)>0, \quad \delta^{\prime \prime \prime}(u)<0 \quad \text { for all } \quad u \geq 0,
$$

and thus

$$
\frac{d}{d v} \delta^{\prime \prime}(u-v) \delta^{\prime}(v)=-\delta^{\prime \prime \prime}(u-v) \delta^{\prime}(v)+\delta^{\prime \prime}(u-v) \delta^{\prime \prime}(v)>0 \quad \text { for all } \quad u \geq v .
$$

For $a<0$ this means that $G_{a}^{\prime \prime \prime}(u) \geq 0$ for $u \geq 0$ if, and only if,

$$
a \leq a^{*}:=\min _{u \geq 0} \frac{u \delta^{\prime \prime \prime}(u)+\delta^{\prime \prime}(u)}{\int_{0}^{u} \delta^{\prime \prime}(u-v) \delta^{\prime}(v) d v+\frac{\delta^{\prime}(u)}{2}} .
$$

A numerical computation shows that $a^{*}=-0.0741 \ldots$

For $a \geq \frac{2}{3}$ it follows from (2.2) that

$$
\frac{1}{a} G_{a}^{\prime \prime \prime}(u) \geq \frac{1}{3}\left(u \delta^{\prime \prime}(u)+\delta^{\prime}(u)\right)-u \delta^{\prime \prime \prime}(u)-\delta^{\prime \prime}(u) .
$$


Elementary considerations show that the right-hand side of this inequality is positive for all $u \geq 0$. Therefore $G_{a}^{\prime \prime \prime}(u)>0$ in $(0, \infty)$ for $a \in\left(-\infty, a^{*}\right] \cup\left[\frac{2}{3}, \infty\right)$, but not for $a \in\left(a^{*}, 0\right)$. Since it has been shown in [4, Thm. 2] that $\xi(x)$ is not completely monotonic for $a \in\left(0, \frac{2}{3}\right)$, the proof of Theorem 1.3 (1) is complete.

Finally, for the proof of Theorem $1.3(2)$, note that the functions $f_{1}(x)$ and $f_{2}(x)$ are merely the function $\xi$ in the special cases $a=\frac{2}{3}$ and $a=1$ and that, as shown in the proof of [4, Cor. 2], the function $f_{3}(x)$ is the Laplace transform of a function $\rho_{3}(u)$, for which $\rho_{3}^{\prime \prime}(u)=u \delta^{\prime \prime}(u), u \in[0, \infty)$, with $\delta(u)$ as defined above. Since it was shown above that $\left(u \delta^{\prime \prime}(u)\right)^{\prime}=u \delta^{\prime \prime \prime}(u)+\delta^{\prime \prime}(u)$ changes sign in $[0, \infty)$, it follows from [6, Thm. 1.3] that $f_{3}$ cannot be completely monotonic of order 3 on $[0, \infty)$.

\section{Proof of Theorem 1.1}

First, note that $f_{\alpha, \beta}(x)=-f_{\beta, \alpha}(x)$ and therefore it will be enough to examine the curvature of $f_{\alpha, \beta}(x)$ in $(0, \infty)$ for $(\alpha, \beta) \in H$.

Next, observe that

$$
f_{\alpha, \beta}^{\prime \prime}(x)=\frac{e^{x} g_{\alpha, \beta}\left(e^{x}\right)}{\left(e^{x}-1\right)^{3}}, \quad x \geq 0 .
$$

Hence, the curvature of $f_{\alpha, \beta}(x)$ in $(0, \infty)$ is completely determined by the sign of $g_{\alpha, \beta}(t)$ in $(1, \infty)$.

Theorem 1.1 now follows from the following four lemmas.

Lemma 3.1. For $\alpha<0$ the function $g_{\alpha}(t)$ is negative in $(1, \infty)$ and for $0<\alpha \leq \frac{1}{2}$ and $\alpha>1$ the function $g_{\alpha}(t)$ is positive in $(1, \infty)$. For $\frac{1}{2}<\alpha<1$ the function $g_{\alpha}(t)$ changes sign in $(1, \infty)$.

Proof. For all $\alpha \in \mathbb{R}$ we have $g_{\alpha}(1)=0$ and

$$
\begin{aligned}
& g_{\alpha}^{\prime}(t)=(\alpha-1)^{2}(\alpha+1) t^{\alpha}+\left(-2 \alpha^{3}+2 \alpha^{2}+\alpha\right) t^{\alpha-1} \\
&+\alpha^{2}(\alpha-1) t^{\alpha-2}-1, \\
& g_{\alpha}^{\prime \prime}(t)= \alpha(\alpha-1) t^{\alpha-3}(t-1)\left(\left(\alpha^{2}-1\right) t-\alpha(\alpha-2)\right), \\
& g_{\alpha}^{\prime \prime \prime}(1)=\alpha(\alpha-1)(2 \alpha-1) .
\end{aligned}
$$

Consequently, for all $\alpha \in \mathbb{R}, g_{\alpha}^{\prime}(1)=g_{\alpha}^{\prime \prime}(1)=0$.

Case $-1 \leq \alpha \leq \frac{1}{2}$ and $1<\alpha$. In this case $g_{\alpha}^{\prime \prime}$ does not vanish in $(1, \infty)$ and thus the sign of $g_{\alpha}^{\prime}$ in $(1, \infty)$ will be equal to the sign of $g_{\alpha}^{\prime \prime \prime}(1)$. For $-1 \leq \alpha<0$ we have $g_{\alpha}^{\prime \prime \prime}(1)<0$, whereas $g_{\alpha}^{\prime \prime \prime}(1)>0$ for $0<\alpha<\frac{1}{2}$ or $1<\alpha$. Therefore, $g_{\alpha}$ is negative in $(1, \infty)$ if $-1 \leq \alpha<0$ and positive if $0<\alpha \leq \frac{1}{2}$ or $1<\alpha$.

Case $\alpha<-1$. In this case $g_{\alpha}^{\prime \prime}$ has exactly one zero $t_{\alpha}$ in $(1, \infty)$. Since $g_{\alpha}^{\prime \prime \prime}(1)<0$, it follows that $g_{\alpha}^{\prime \prime}<0$ in $(1, \infty)$ if and only if $1<t<t_{\alpha}$. Since $g_{\alpha}^{\prime}(t) \rightarrow-1$ as $t \rightarrow \infty$, this shows that $g_{\alpha}^{\prime}$ is negative in $(1, \infty)$. Hence, for $\alpha<-1, g_{\alpha}$ is negative in $(1, \infty)$.

Case $\frac{1}{2}<\alpha<1$. In this case we have $g_{\alpha}^{\prime \prime \prime}(1)<0$ and thus $g_{\alpha}(t)<0$ for $t>1$ close to 1 . Since obviously $g_{\alpha}(t) \rightarrow \infty$ as $t \rightarrow \infty$, the proof of the lemma is complete.

Lemma 3.2. For $(\alpha, \beta) \in B \backslash \Gamma_{1}$, the sign of $g_{\alpha, \beta}(t)$ changes on $(1, \infty)$.

Proof. Since $g_{1, \beta}(t)=-g_{\beta}(t)$, the case $\alpha=1$ of our assertion follows from Lemma 3.1. For the other $(\alpha, \beta)$ in question we have $\alpha \neq 1$ and $\alpha>\beta$ and thus

$$
\lim _{t \rightarrow \infty} t^{-(1+\alpha)} g_{\alpha, \beta}(t)=(1-\alpha)^{2}>0 .
$$


It will therefore be enough to show that $g_{\alpha, \beta}(t)$ takes negative values in $(1, \infty)$ for $(\alpha, \beta) \in B$ with $\alpha \neq 1$.

We have $g_{\alpha, \beta}^{(n)}(1)=0$ for $n=0,1,2$ and

$$
\frac{g_{\alpha, \beta}^{(3)}(1)}{\alpha-\beta}=\varepsilon_{1}(\alpha, \beta)
$$

Consequently, for

$$
(\alpha, \beta) \in\left\{(\alpha, \beta): \beta<\alpha, \varepsilon_{1}(\alpha, \beta)<0\right\},
$$

$g_{\alpha, \beta}(t)$ takes negative values in $(1, \infty)$ and it only remains to show that the same is true for $(\alpha, \beta)$ in the triangle $\left\{(\alpha, \beta): \frac{1}{2}<\beta<\alpha<1\right\}$.

To that end, fix a $\beta \in\left(\frac{1}{2}, 1\right)$ and observe that by Lemma 3.1 there is a $t^{*} \in(1, \infty)$ such that $g_{\beta}\left(t^{*}\right)=0$. Our claim will follow once we have shown that the function $h(\alpha):=g_{\alpha}\left(t^{*}\right), \alpha \in\left(\frac{1}{2}, 1\right)$, is negative for all $\alpha \in(\beta, 1)$, since

$$
g_{\alpha, \beta}\left(t^{*}\right)=g_{\alpha}\left(t^{*}\right)-g_{\beta}\left(t^{*}\right)=h(\alpha) .
$$

We calculate

$\left(t^{*}\right)^{1-\alpha} h^{\prime}(\alpha)=2\left(t^{*}-1\right)\left(\alpha\left(t^{*}-1\right)-t^{*}\right)+\log t^{*}\left(\alpha^{2}\left(t^{*}-1\right)^{2}-2 t^{*} \alpha\left(t^{*}-1\right)+t^{*}\left(t^{*}+1\right)\right)$, and thus $h^{\prime}(\alpha)$ vanishes for those $\alpha$ for which the rational function

$$
r(\alpha):=\frac{\alpha^{2}\left(t^{*}-1\right)^{2}-2 t^{*} \alpha\left(t^{*}-1\right)+t^{*}\left(t^{*}+1\right)}{\alpha\left(t^{*}-1\right)-t^{*}}
$$

cuts the horizontal $\alpha \mapsto 2\left(1-t^{*}\right) / \log t^{*}$. It is straightforward to verify that, in $\left(\frac{1}{2}, 1\right), r(\alpha)$ has no pole and $r^{\prime}(\alpha)$ has exactly one zero and hence $h$ can have at most two local extrema in $\left(\frac{1}{2}, 1\right)$.

Now, suppose that $h^{\prime}(\beta)>0$. Then, since $h(1)=0$ and

$$
h^{\prime}(1)=\log t^{*}\left(t^{*}+1\right)+2\left(1-t^{*}\right)>0
$$

for all $t^{*} \in(1, \infty), h$ must have at least two local extrema in $(\beta, 1)$. On the other hand, $4 \sqrt{t^{*}} h\left(\frac{1}{2}\right)=\left(\sqrt{t^{*}}-1\right)^{4}>0$ and hence $h(\alpha)$ has to have at least one local minimum in $\left(\frac{1}{2}, \beta\right)$. But $h(\alpha)$ can have at most two local extrema in $\left(\frac{1}{2}, 1\right)$ and therefore $h^{\prime}(\beta)<0$. If now $h(\alpha)>0$ would hold for an $\alpha \in(\beta, 1)$, then, since $h(1)=0$ and $h^{\prime}(1)>0, h(\alpha)$ would have to have more than two local extrema in $(\beta, 1)$. Thus, we must have $h(\alpha)<0$ for all $\alpha \in(\beta, 1)$, and the proof of the lemma is complete.

Lemma 3.3. For $(\alpha, \beta) \in H \backslash D$ we have $g_{\alpha, \beta}(t) \geq 0$ in $(1, \infty)$, and for $0 \leq \beta \leq \frac{1}{2}$ the function $g_{1, \beta}(t)$ is nonpositive in $(1, \infty)$.

Proof. The case $\alpha=\beta$ is trivial and since $g_{\alpha, 1}(t)=g_{\alpha}(t)$ and $g_{1, \beta}(t)=-g_{\beta}(t)$, the cases $\alpha=1$ and $\beta=1$ of our assertion follow from Lemma 3.1

In order to prove the lemma also for the other $(\alpha, \beta)$ in question, set

$$
h_{\alpha}(t):=(1-\alpha)^{2} t^{2}+\left(1+2 \alpha-2 \alpha^{2}\right) t+\alpha^{2}, \quad \alpha, t \in \mathbb{R} .
$$

The parabola $h_{\alpha}(t)$ opens upward for all $\alpha \in \mathbb{R}$, its discriminant is nonnegative exactly for $\frac{1}{2}(1-\sqrt{2}) \leq \alpha \leq \frac{1}{2}(1+\sqrt{2})<\frac{3}{2}$, and $h_{\alpha}^{\prime}(1)>0$ exactly if $\alpha<\frac{3}{2}$. Hence, $h_{\alpha}(t)>0$ for all $\alpha \in \mathbb{R}$ and $t>1$, and therefore $g_{\alpha, \beta}(t) \geq 0$ is equivalent to

$$
\log h_{\alpha}(t)-\log h_{\beta}(t) \geq(\beta-\alpha) \log t
$$




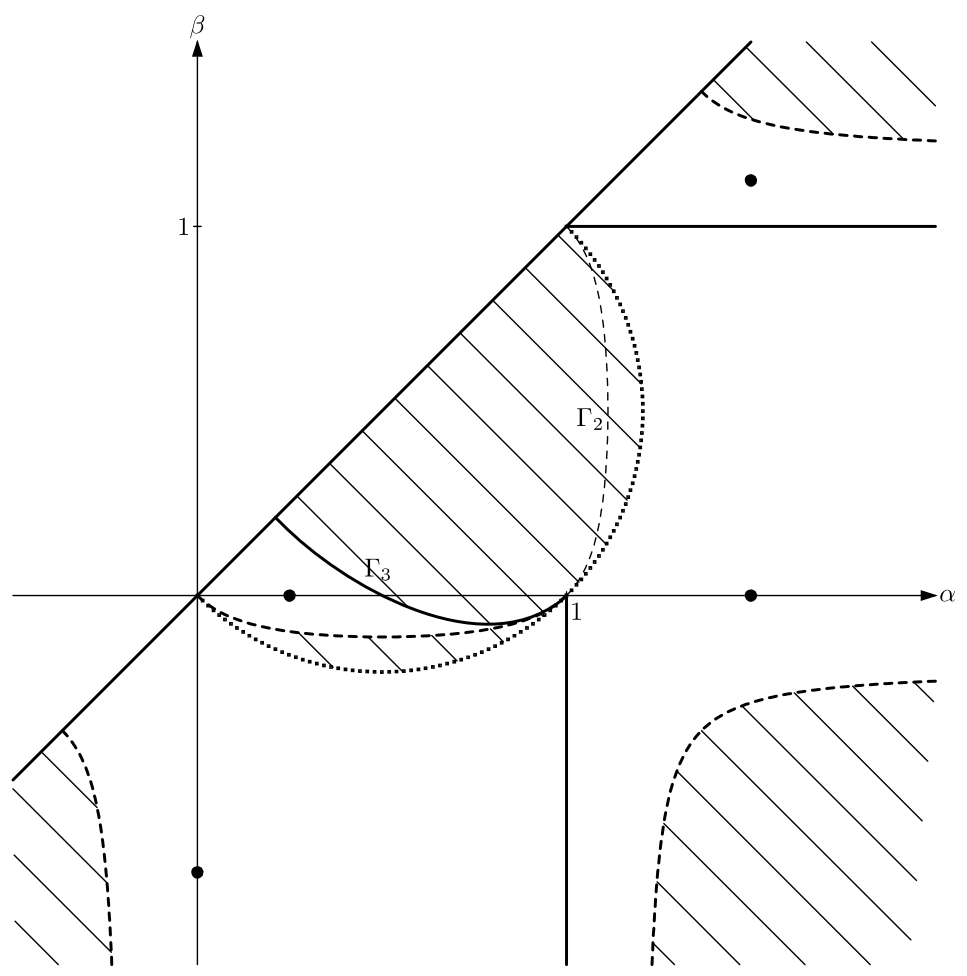

Figure 3 . The sets $P$ and $N$. The interior of $P$ is hatched. The bold curves are $\partial N$. The dotted arc describes the set $H \cap\{(\alpha, \beta)$ : $\left.\varepsilon_{3}(\alpha, \beta)=0\right\}$. The dashed curves describe the set $H \cap\{(\alpha, \beta)$ : $\left.\varepsilon_{2}(\alpha, \beta)=0\right\}$. The four dots describe the location of the points $q_{1}, \ldots, q_{4}$.

Since this inequality holds for all $(\alpha, \beta) \in \mathbb{R}$ when $t=1$, it will suffice to prove that

$$
\frac{h_{\alpha}^{\prime}(t)}{h_{\alpha}(t)}-\frac{h_{\beta}^{\prime}(t)}{h_{\beta}(t)} \geq \frac{\beta-\alpha}{t}
$$

or

$$
t h_{\alpha}^{\prime}(t) h_{\beta}(t)-t h_{\beta}^{\prime}(t) h_{\alpha}(t)+(\alpha-\beta) h_{\alpha}(t) h_{\beta}(t) \geq 0 \text { for } t>1 \text {. }
$$

The left-hand side of the latter inequality is equal to $(\alpha-\beta)(1-t)^{2} p_{\alpha, \beta}(t)$, where

$$
p_{\alpha, \beta}(t)=t^{2}(1-\alpha)^{2}(1-\beta)^{2}+t\left(\beta(\beta-1)+\left(\alpha^{2}-\alpha\right)(1-2 \beta(\beta-1))\right)+\alpha^{2} \beta^{2},
$$

so that (3.1) is equivalent to

$$
p_{\alpha, \beta}(t) \geq 0 \text { for } t>1 .
$$

The discriminant of the parabola (in the following we will always assume that $\alpha \neq 1$ and $\beta \neq 1) p_{\alpha, \beta}(t)$ is given by $-\varepsilon_{2}(\alpha, \beta) \varepsilon_{3}(\alpha, \beta)$. Since $p_{\alpha, \beta}(t)$ opens upward, it therefore follows that the nonnegativity of $p_{\alpha, \beta}(t)$ in $(1, \infty)$ only remains to be verified for $(\alpha, \beta) \in N:=H \backslash P$, where (cf. Figure 3)

$$
P:=D \cup\left\{(\alpha, \beta): \varepsilon_{2}(\alpha, \beta) \varepsilon_{3}(\alpha, \beta) \geq 0\right\} \cup\{(1, \beta): \beta \in \mathbb{R}\} \cup\{(\alpha, 1): \alpha \in \mathbb{R}\} .
$$


A straightforward computation shows that if $p_{\alpha, \beta}(t)$ has a zero at 1 , then $\varepsilon_{1}(\alpha, \beta)$ $=0$ must hold. Since $\left\{(\alpha, \beta): \varepsilon_{1}(\alpha, \beta)=0\right\} \cap H$ is contained in $\bar{D}$, a continuity argument yields that the number of zeros of $p_{\alpha, \beta}(t)$ in $[1, \infty)$ is constant in each component of $N$.

It is easy to verify that $N$ consists of exactly 4 components and that no two of the points $q_{1}:=\left(\frac{1}{4}, 0\right), q_{2}:=\left(\frac{3}{2}, 0\right), q_{3}:=\left(0,-\frac{3}{4}\right)$ and $q_{4}:=\left(\frac{3}{2}, \frac{9}{8}\right)$ lie in the same component of $N$ (cf. Figure 3i). Since one readily sees that $p_{q_{j}}(t)$ has no zeros in $(1, \infty)$ for $j=1, \ldots, 4$, it follows that $p_{\alpha, \beta}(t)$ is positive in $(1, \infty)$ for all $(\alpha, \beta) \in N$.

The proof of the lemma is complete.

Lemma 3.4. The function $f_{\alpha, \beta}(x)$ is convex on $(0, \infty)$ if $(\alpha, \beta) \in C_{\text {conv. For }}$ $(\alpha, \beta) \in C \backslash C_{\text {conv }}$ and $(\alpha, \beta) \in \Gamma_{1}$ the sign of $f_{\alpha, \beta}^{\prime \prime}(x)$ changes on $(0, \infty)$.

Proof. It follows from the proof of Lemma 3.3 that $g_{\alpha, \beta}(t)$ has a critical point $t$ in $(1, \infty)$ if, and only if, $t$ is a zero of $p_{\alpha, \beta}(t)$. Furthermore, since one easily checks that $\alpha, \beta \neq 1$ and $\varepsilon_{1}(\alpha, \beta) \neq 0$ for all $(\alpha, \beta)$ in the connected set $C$, the proof of Lemma 3.3 also shows that the number of zeros of $p_{\alpha, \beta}(t)$ in $[1, \infty)$ is constant in $C$. It is readily verified that $q_{5}:=\left(\frac{11}{10}, \frac{1}{2}\right) \in C$ (cf. Figure 21) and that $p_{q_{5}}(t)$ has exactly two zeros in $(1, \infty)$. Hence, for $(\alpha, \beta) \in C, g_{\alpha, \beta}(t)$ has exactly two local extrema in $(1, \infty)$. Since $\beta<\alpha \neq 1$ in $C$, it follows from the proof of Lemma 3.2 that $g_{\alpha, \beta}(t)$ is positive for all $t$ large enough. Since moreover $g_{\alpha, \beta}(1)=0$ for all $(\alpha, \beta) \in \mathbb{R}^{2}$, the largest one, say $t^{*}$, of the critical points of $g_{\alpha, \beta}(t)$ in $[1, \infty)$ must be a local minimum of $g_{\alpha, \beta}(t)$, and $g_{\alpha, \beta}(t)$ will be nonnegative in $(1, \infty)$ if, and only if, $g_{\alpha, \beta}\left(t^{*}\right) \geq 0$. $t^{*}$ must be the largest zero of $p_{\alpha, \beta}(t)$ and can thus be calculated to be $t^{*}(\alpha, \beta)$.

The set $\Gamma_{1}$ belongs to the boundary of both $C$ and $E$, where $E$ is the open bounded set that has the Jordan curve $\Gamma_{1} \cup\left\{(1, \beta): 0 \leq \beta \leq \frac{1}{2}\right\}$ as its boundary ( $E$ is shaded in Figure 22). The point $q_{6}:=\left(\frac{101}{100}, \frac{1}{4}\right)$ lies in $E$ and $p_{q_{6}}(t)$ has exactly one zero in $(1, \infty)$. Hence, on $\Gamma_{1}$, at least one of the zeros of $p_{\alpha, \beta}(t)$ is equal to 1 . Since $\varepsilon_{2}(\alpha, \beta) \varepsilon_{3}(\alpha, \beta) \neq 0$ for $(\alpha, \beta) \in \Gamma_{1}$, the function $p_{\alpha, \beta}(t)$ cannot have a double zero at $t^{*}(\alpha, \beta)$ on $\Gamma_{1}$. Therefore $t^{*}(\alpha, \beta)$ is the only critical point of $g_{\alpha, \beta}(t)$ in $(1, \infty)$ when $(\alpha, \beta) \in \Gamma_{1}$. Since $g_{\alpha, \beta}(1)=0$ and $g_{\alpha, \beta}(t)>0$ for large $t$, this means that we must have $g_{\alpha, \beta}\left(t^{*}(\alpha, \beta)\right)<0$ for $(\alpha, \beta) \in \Gamma_{1}$.

\section{REFERENCES}

1. J. Dubourdieu, Sur un théorème de M. S. Bernstein relatif à la transformation de LaplaceStieltjes. Compositio Math. 7 (1939), 96-111. MR0000436 (1:73h)

2. H. van Haeringen, Completely monotonic and related functions. J. Math. Anal. Appl. 204 (1996), 389-408. MR 1421454 (97j:26010)

3. KETpic for Maple. Available online at www.kisarazu.ac.jp/ ${ }^{\sim}$ masa/math/.

4. S. Koumandos, Monotonicity of some functions involving the Gamma and Psi functions, Math. Comp. 77 (2008), 2261-2275. MR 2429884

5. S. Koumandos and M. Lamprecht, On a conjecture for trigonometric sums and starlike functions II, submitted.

6. S. Koumandos and H. L. Pedersen, Completely monotonic functions of positive order and asymptotic expansions of the logarithm of Barnes double gamma function and Euler's gamma function, J. Math. Anal. Appl. 355(1) (2009) 33-40.

7. S. Koumandos and S. Ruscheweyh, Positive Gegenbauer polynomial sums and applications to starlike functions. Constr. Approx. 23 (2006), no. 2, 197-210. MR2186305 (2007b:42001)

8. S. Koumandos and S. Ruscheweyh, On a conjecture for trigonometric sums and starlike functions. J. Approx. Theory 149, (2007), no. 1, 42-58. MR2371613 (2009f:42001)

9. F. Qi, Bounds for the ratio of two gamma function, arXiv:0902.2514v1 [math.CA]. 
10. S. Y. Trimble, J. Wells, and F. T. Wright, Superadditive functions and a statistical application, SIAM J. Math. Anal. 20 no. 5 (1989), 1255-1259. MR.1009357(91a:26019)

11. D. V. Widder, The Laplace transform. Princeton University Press, Princeton, 1946. MR0005923 (3:232d)

Department of Mathematics and Statistics, The University of Cyprus, P. O. Box 20537, 1678 Nicosia, Cyprus

E-mail address: skoumand@ucy.ac.cy

Department of Mathematics and Statistics, The University of Cyprus, P. O. Box 20537, 1678 Nicosia, Cyprus

E-mail address: martin@ucy.ac.cy 